\title{
Tratamento com Dexametasona reduz atividade exploratória de ratos Wistar jovens
}

\author{
Dexamethasone treatment reduces the exploratory activity \\ of young Wistar rats
}

\section{El tratamiento con dexametasona reduce la actividad exploratoria de ratas Wistar jóvenes}

\author{
Camila da Silva Almeida ${ }^{1}$, Ana Paula Assis ${ }^{2}$, Marcella Oliveira \\ Monteiro ${ }^{3}$, Valéria Ernestânia Chaves ${ }^{4}$, Maira de Castro Lima ${ }^{5}$
}

1.Graduanda em Farmácia, Universidade Federal de São João Del-Rei, Divinópolis-MG, Brasil. https://orcid.org/0000-0001-6249-5182

2.Bioquímica, Mestre, Universidade de São Paulo, Ribeirão Preto-SP, Brasil. https://orcid.org/0000-0002-5738-9374

3.Graduanda em Farmácia, Universidade Federal de São João Del-Rei, Divinópolis-MG, Brasil. https://orcid.org/0000-0002-9920-4737

4.Nutricionista, Doutora, Universidade Federal de São João Del-Rei, Divinópolis-MG, Brasil. https://orcid.org/0000-0003-4026-8565

5.Fisioterapeuta, Doutora, Universidade Federal de São João Del-Rei, Divinópolis-MG, Brasil. https://orcid.org/0000-0002-5403-4033

\section{Resumo}

Introdução. Dexametasona (DEX) é um glicocorticóide sintético com ação anti-inflamatória utilizado no tratamento de muitas doenças como artrite reumatoide, asma, alergias e doenças autoimunes. Estudos mostram que a DEX pode causar deficits cognitivos em animais, podendo estar relacionada com a redução na atividade exploratória, prejuízo na aprendizagem e memória espacial e aumento da ansiedade. Objetivo. O presente estudo investigou os efeitos da DEX sobre atividade exploratória, ansiedade e memória espacial em ratos jovens. Método. Ratos Wistar machos foram tratados com fosfato de dexametasona nas dosagens de 1 e 10 $\mathrm{mg} / \mathrm{Kg} /$ dia durante 7 dias. No $5^{\circ}$ dia de tratamento foi realizado o teste de campo aberto (CA) para avaliação da atividade exploratória; no $6^{\circ}$ dia foi realizado o teste de labirinto em cruz elevado (LCE) para avaliação da ansiedade e no $7^{\circ}$ dia foi realizado o teste de labirinto em $T$ elevado (LTE) para avaliação da memória espacial. Resultados. No CA, houve redução de $48 \%$ no número total de quadrantes explorados no grupo DEX $10 \mathrm{mg} / \mathrm{Kg}, 46 \%$ e $56 \%$ de redução no número de quadrantes centrais explorados nos grupos DEX 1 e $10 \mathrm{mg} / \mathrm{Kg}$, respectivamente, e $40 \%$ de redução no número de elevações no grupo DEX $(10 \mathrm{mg} / \mathrm{Kg})$, indicando que a DEX diminuiu a atividade exploratória dos animais tratados. Nos testes LCE e LTE não houve diferenças entre os grupos experimentais, indicando que a DEX não interferiu no comportamento de ansiedade e memória espacial. Conclusão. Nas condições experimentais deste estudo, a DEX diminuiu atividade exploratória mas não alterou ansiedade e memória espacial.

Unitermos. Cognição; Glicocorticoide; Análise Comportamental Aplicada

\footnotetext{
Abstract

Introduction. Dexamethasone (DEX) is a synthetic glucocorticoid with anti-inflammatory action used in the treatment of many diseases such as rheumatoid arthritis, asthma, allergies, and autoimmune diseases. Studies show that DEX can cause cognitive deficits in animals, which may result in reduced exploratory activity, impaired learning and spatial memory and increased anxiety. Objective. This study investigated the effects of DEX on exploratory activity, anxiety and spatial memory in young rats. Method. Young male Wistar rats were treated with dexamethasone phosphate at doses of 1 and $10 \mathrm{mg} / \mathrm{kg} / \mathrm{day}$ for 7 days. On the
} 
5th day of treatment, the open field test (OF) was performed to evaluate exploratory activity; on the 6th day, the elevated plus maze test (EPM) was performed to evaluate anxiety, and on the 7th day, the elevated T maze test (ETM) was performed to evaluate spatial memory. Results. In OF there was a $48 \%$ reduction in the number of total quadrants explored in the DEX group $10 \mathrm{mg} / \mathrm{kg}, 46 \%$ and $56 \%$ reduction in the number of central quadrants explored in DEX 1 and $10 \mathrm{mg} / \mathrm{kg}$ groups, respectively, and $40 \%$ reduction in the number of elevations in the DEX $10 \mathrm{mg} / \mathrm{kg}$ group, indicating that DEX decreased the exploratory activity on treated animals. In the EPM and ETM tests, there were no differences between the experimental groups, indicating that DEX did not interfere in the anxiety behavior and spatial memory. Conclusion. In the experimental conditions of this study, DEX decreased exploratory activity but did not alter anxiety behavior and spatial memory.

Keywords. Cognition; Glucocorticoid; Applied Behavior Analysis

\section{Resumen}

Introducción. Dexametasona (DEX) es un glucocorticoide sintético con acción antiinflamatoria utilizado en el tratamiento de muchas enfermedades como artritis reumatoide, asma, alergias y enfermedades autoinmunes. Estudios muestran que la DEX puede causar déficits cognitivos en animales, puede resultar en actividad exploratoria reducida, aprendizaje y memoria espacial deficientes y un aumento de la ansiedad. Objetivo. Este estudio investigó los efectos de DEX sobre actividad exploratoria, ansiedad y memoria espacial en ratas jóvenes. Método. Se trataron ratas Wistar macho jóvenes con fosfato de dexametasona a dosis de 1 y $10 \mathrm{mg} / \mathrm{kg} /$ día durante 7 días. Al quinto día, se realizó la prueba de campo abierto (CA) para evaluar la actividad exploratoria; en el sexto día, se realizó la prueba de laberinto cruz elevado (LCE) para evaluar la ansiedad, y en el séptimo día se realizó la prueba de laberinto en T elevado (LTE) para evaluar la memoria espacial. Resultados. En OF hubo una reducción del $48 \%$ en el número de cuadrantes totales explorados en el grupo DEX $10 \mathrm{mg} / \mathrm{kg}, 46 \%$ y $56 \%$ de reducción en el número de cuadrantes centrales explorados en los grupos DEX 1 y 10 $\mathrm{mg} / \mathrm{kg}$, respectivamente, y reducción del $40 \%$ en el número de elevaciones en el grupo DEX $10 \mathrm{mg} / \mathrm{kg}$, lo que indica que DEX disminuyó la actividad exploratoria en los animales tratados. En las pruebas LCE y LTE, no hubo diferencias entre los grupos experimentales, lo que indica que DEX no interfirió en ansiedad y memoria espacial. Conclusión. En las condiciones experimentales de este estudio, DEX disminuyó la actividad exploratoria pero no alteró el comportamiento de ansiedad y la memoria espacial.

Palabras clave. Cognición; Glucocorticoide; Análisis de comportamiento aplicado

Trabalho realizado na Universidade Federal de São João Del-Rei, Campus Centro-Oeste Dona Lindu, Divinópolis-MG, Brasil.

\section{INTRODUÇÃO}

Os glicocorticoides são hormônios esteroidais utilizados em áreas da imunologia, dermatologia, reumatologia e alergologia. Em 1949, foi relatado, pela primeira vez, a eficácia dos glicocorticoides como agentes anti-inflamatórios para o tratamento da artrite reumatoide. Há muitos anos estes medicamentos são empregados em uma variedade de 
condições clínicas com manifestações inflamatórias e alérgicas ${ }^{1-3}$.

A todo momento os organismos vivos são desafiados por estímulos estressores, sendo eles internos ou externos. Estes estímulos induzem a liberação de hormônios, tais como os glicocorticoides, que em desequilíbrio podem afetar negativamente a função cognitiva do animal ${ }^{4}$. Uma vez que os glicocorticoides possuem características lipofílicas, são capazes de atravessar a barreira hematoencefálica, podendo se ligar ao seu receptor e desencadear várias respostas no sistema nervoso ${ }^{5}$.

A Dexametasona (DEX) é um glicocorticoide sintético com propriedades anti-inflamatórias e imunossupressoras e, apresenta forte afinidade aos receptores de glicocorticoides. Foi demonstrado que a administração de DEX em períodos iniciais da vida do animal resulta em alterações na plasticidade sináptica do hipocampo, podendo interferir na formação de memórias ${ }^{6,7}$. O modelo animal é útil para fins de pesquisa, visto que as alterações plásticas que ocorrem no cérebro humano podem ser elucidadas em estudos experimentais ${ }^{8}$.

A administração de DEX nos dias 1, 2 e 3 pós-natais nas doses de 0,5 $\mathrm{g} / \mathrm{g}, 0,3 \mu \mathrm{g} / \mathrm{g}$ e $0,1 \mu \mathrm{g} / \mathrm{g}$, respectivamente, promoveu uma redução no comportamento de ansiedade dos animais tratados, mas não apresentou efeitos sobre a atividade exploratória dos animais adultos, avaliados no $90^{\circ}$ dia pós-natal ${ }^{9}$. Por outro lado, a exposição pré-natal a DEX na dose de $50 \mu \mathrm{g} / \mathrm{g}$ resultou em um aumento no 
comportamento relacionado à ansiedade em prole masculina adulta, na $10^{\mathrm{a}}$ semana pós-natal ${ }^{10}$.

Em outro estudo, a DEX na dose de $1 \mathrm{mg} / \mathrm{Kg}$ não foi capaz de alterar significativamente o comportamento de ansiedade, visto que não houve diferença no teste de labirinto em cruz elevado (LCE). Por outro lado, o tratamento com $5 \mathrm{mg} / \mathrm{kg}$ ou $10 \mathrm{mg} / \mathrm{kg}$ de DEX durante 1 semana induziu um comportamento de ansiedade aumentado ${ }^{11}$.

Com base em evidências científicas da influência da DEX sobre função cognitiva e emocional e a controvérsia de resultados em estudos experimentais em relação às doses, tempo de tratamento e período de vida dos animais tratados, o objetivo deste estudo foi investigar os efeitos da DEX, nas doses de $1 \mathrm{mg} / \mathrm{kg}$ e $10 \mathrm{mg} / \mathrm{kg}$, sobre os padrões comportamentais, atividade exploratória, ansiedade e memória espacial, em ratos Wistar machos jovens.

\section{MÉTODO}

\section{Animais Experimentais e Administração de Dexametasona}

Foram utilizados ratos Wistar machos jovens, com idade entre 5 e 6 semanas de vida e peso inicial entre 125 e 165 gramas, provenientes do biotério Central da UFSJ e mantidos no biotério setorial do Laboratório de Fisiologia no Campus Centro-Oeste Dona Lindu. Os ratos foram alojados em gaiolas coletivas de polietileno, cada uma contendo quatro animais, à temperatura de $22 \pm 2{ }^{\circ} \mathrm{C}$ e em ciclo de claroescuro de 12 horas. Os animais foram divididos em três grupos: DEX 1mg/kg, DEX $10 \mathrm{mg} / \mathrm{kg}$ e controle. Aqueles do 
grupo DEX foram tratados com uma injeção diária, via intraperitoneal, com $1 \mathrm{mg} / \mathrm{kg}$ ou $10 \mathrm{mg} / \mathrm{kg}$ de fosfato de dexametasona (Sigma ${ }^{\circledR}$ ) durante 7 dias. Animais do grupo controle receberam uma injeção de solução salina $0,9 \%$ de mesmo volume, pelo mesmo período de tempo. Os ratos foram eutanasiados no oitavo dia de experimento. Todos os procedimentos experimentais foram aprovados pela Comissão de Ética no Uso de Animais (CEUA) da UFSJ, protocolo experimental 016/2017.

\section{Testes comportamentais}

Os testes comportamentais foram realizados no $5^{\circ}, 6^{\circ}$ e 70 dias de tratamento, após às 18 horas, devido ao ciclo circadiano do animal que apresenta um período de vigília noturno. Foram utilizados os testes de campo aberto (CA), labirinto em cruz elevado (LCE) e teste de labirinto em T elevado (LTE). O delineamento experimental está representado na Figura 1.

O aparato de teste e os objetos utilizados foram limpos com álcool $70 \%$ antes cada sessão. Todos os testes foram filmados para posterior análise do comportamento animal por avaliadores que desconheciam os grupos experimentais dos ratos avaliados.

\section{Teste de Campo Aberto}

Foi realizado no $5^{0}$ dia de tratamento, para avaliar atividade exploratória espontânea dos animais, em uma caixa de acrílico $(70 \mathrm{~cm} \times 70 \mathrm{~cm} \times 40 \mathrm{~cm})$ leitoso, com uma das 
laterais transparente e o fundo da caixa dividido em vinte e cinco quadrantes.

Figura 1. Delineamento experimental: tratamento com Dexametasona durante 7 dias, realização do teste de campo aberto no $5^{\circ}$ dia, labirinto em cruz elevado no $6^{\circ}$ dia e labirinto em $T$ elevado no $7^{\circ}$ dia, seguido pela eutanásia do animal no $8^{\circ}$ dia de experimento.

Procedimento experimental

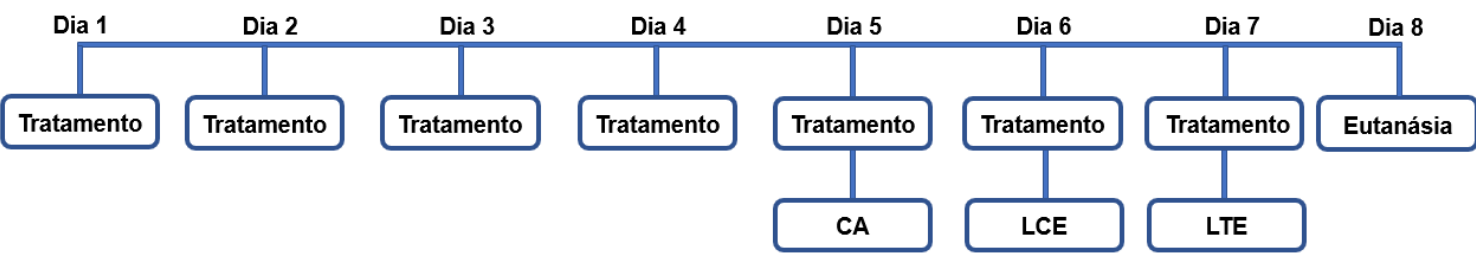

O teste foi realizado por 10 minutos e os animais foram colocados sempre no mesmo quadrante inicial. Os parâmetros avaliados foram os números de mudança de quadrantes, visitas aos quadrantes centrais e elevações do corpo, além do tempo do comportamento de auto limpeza. O número de animais em cada grupo para o teste de CA foi: Controle $\mathrm{n}=15$, DEX $1 \mathrm{mg} / \mathrm{Kg} \mathrm{n}=15$ e DEX $10 \mathrm{mg} / \mathrm{Kg} \mathrm{n}=14$.

\section{Teste de Labirindo em Cruz Elevado}

O comportamento de ansiedade foi avaliado no $6^{\circ}$ dia de tratamento, através do teste de LCE. O aparato de teste consiste em dois braços fechados e dois abertos, horizontais, dispostos perpendicularmente, posicionados 50 centímetros acima do chão. Os animais foram posicionados na plataforma central, $(10 \mathrm{~cm} \times 10 \mathrm{~cm})$, no meio da ligação dos quatro braços, para iniciar o teste que durou 10 minutos. Foi 
avaliada a locomoção do rato, mensurando-se o tempo de permanência e frequência de visita aos braços abertos, à área central e aos braços fechados. Um aumento no tempo de permanência nos braços fechados comparados com o tempo de permanência do grupo controle, refere-se ao comportamento de ansiedade aumentado. O número de animais em cada grupo para o teste de LCE foi: Controle $\mathrm{n}=13$, DEX $1 \mathrm{mg} / \mathrm{Kg} \mathrm{n}=15$ e DEX $10 \mathrm{mg} / \mathrm{Kg} \mathrm{n}=11$.

\section{Teste de Labirinto em T Elevado}

A memória espacial foi avaliada no 70 dia de tratamento. O aparato de teste foi o mesmo utilizado para o LCE, mas com um dos braços fechados bloqueado, assumindo o formato em T. A habituação do animal ao aparato aconteceu no dia anterior na realização do teste de LCE. Foram realizados dois treinos e um teste, de cinco minutos cada, com um intervalo de 30 minutos entre eles.

Nas sessões denominadas treinos 1 e 2, um objeto foi colocado no braço aberto esquerdo e os animais puderam explorar o aparato. Após os treinos, foi realizado o teste em que o objeto foi colocado no braço contralateral do aparato. Foi mensurado o tempo de exploração do objeto e o tempo de permanência e o número de entradas no braço onde estava o objeto nos treinos. De acordo com os parâmetros do teste, a memória espacial do animal não é comprometida quando há um aumento no tempo de permanência e no número de entradas no braço onde estava o objeto durante o treino (esquerdo) e/ou uma redução no tempo de 
exploração do objeto.

O número de animais em cada grupo para o teste de LTE foi: Controle $\mathrm{n}=10$, DEX $1 \mathrm{mg} / \mathrm{Kg} \mathrm{n}=11$ e DEX $10 \mathrm{mg} / \mathrm{Kg}$ $\mathrm{n}=9$.

\section{Análise Estatística}

Os resultados foram expressos em média \pm erro padrão da média. As diferenças entre os grupos foram analisadas utilizando o One Way ANOVA, post hoc de Tukey, com $P<0,05$ para nível de significância. Foi realizado o teste de normalidade que mostrou que a distribuição dos dados é normal.

\section{RESULTADOS E DISCUSSÃO \\ Teste de Campo Aberto}

Os animais tratados com DEX $(10 \mathrm{mg} / \mathrm{Kg})$ apresentaram uma redução de aproximadamente $48 \%$ no número de quadrantes visitados (Figura $2 \mathrm{~A}$ ). Ao analisar a visita aos quadrantes centrais, observou-se uma redução de 46 e 56\%, nos grupos tratados com DEX (1 e $10 \mathrm{mg} / \mathrm{Kg})$, respectivamente (Figura $2 \mathrm{~B}$ ). Houve uma redução de $40 \%$ das elevações corporais dos ratos tratados com DEX (10mg/Kg; Figura 2C). Não houve diferença com relação ao tempo do comportamento de auto limpeza entre os grupos. 
Figura 2. Efeitos do tratamento com Dexametasona sobre a atividade exploratória espontânea. (A) visita aos quadrantes totais; (B) visita aos quadrantes centrais; (C) número de elevações corporais. $* p<0,05$ vs. controle. Controle $n=15, D E X 1 \mathrm{mg} / \mathrm{Kg}$ $\mathrm{n}=15$ e DEX $10 \mathrm{mg} / \mathrm{Kg} \mathrm{n}=14$.

\section{Campo Aberto}

A

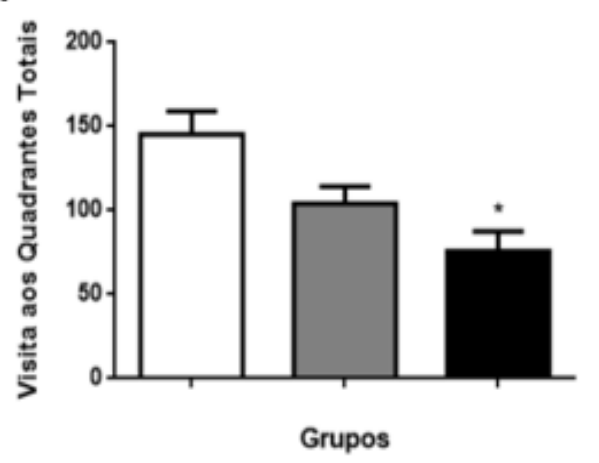

C
B

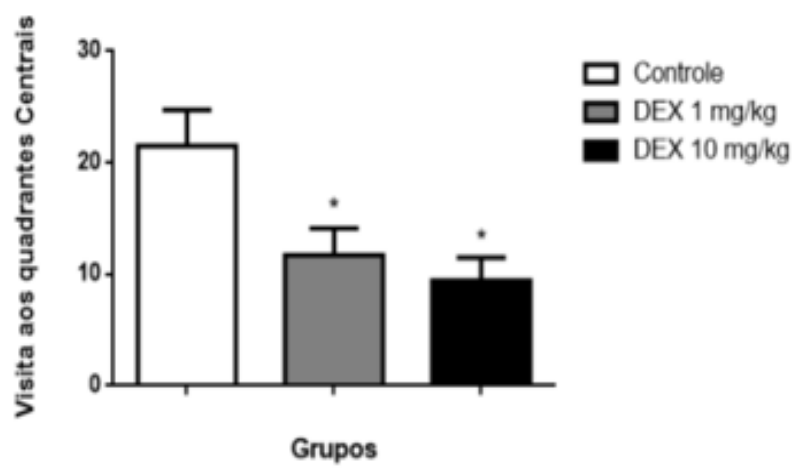

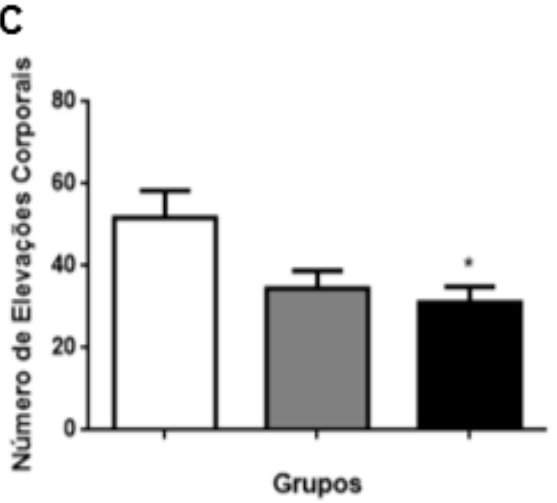

Os resultados do presente estudo corroboram com os achados de Yilmaz et $a l^{12}$ que demonstraram que em ratos adultos a administração de DEX $10 \mathrm{mg} / \mathrm{Kg}$ durante 9 dias, reduziu o número de quadrantes totais percorridos e 0 número de elevações corporais quando comparados com o grupo controle, indicando uma diminuição no comportamento exploratório ${ }^{12}$. Observa-se que a dose é a mesma utilizada nesse trabalho e o tempo de tratamento com a DEX é bastante parecido, favorecendo respostas biológicas semelhantes. 
Outros trabalhos mostraram que não há mudança na atividade exploratória espontânea dos animais utilizando-se doses de vinte até cem vezes menores que a de $10 \mathrm{mg} / \mathrm{kg}$. Em um tratamento neonatal com DEX nas doses de $0,5 \mu \mathrm{g} / \mathrm{g}$, $0,3 \mu \mathrm{g} / \mathrm{g}$ e $0,1 \mu \mathrm{g} / \mathrm{g}$, durante os dias 1,2 e 3 de vida, respectivamente, demonstrou que o tratamento não afetou a distância percorrida no CA e não resultou em diferenças na atividade exploratória central e periférica durante os quatro dias consecutivos de teste, quando avaliado no $50^{\circ}$ dia de vida, indicando que o comportamento exploratório no CA não foi influenciado pelo tratamento com $\mathrm{DEX}^{13}$. A plasticidade neural é intensa no período pós-natal, sendo um período crítico para ação biológica de agentes externos, entretanto a dose é importante na indução de potenciais mudanças.

Uma dose de $1,5 \mathrm{mg} / \mathrm{kg}$, em um período pós-natal um pouco maior, 5 a 10 dias, não resultou em diferenças entre os grupos no total de quadrantes percorridos, bem como no número de quadrantes periféricos no $C A$. No entanto, o número de quadrantes centrais percorridos foi maior no grupo DEX do que no grupo controle. Esse aumento de visitas aos quadrantes centrais também pode ser interpretado como indicativo de um animal com ansiedade reduzida ${ }^{14}$.

\section{Teste de Labirinto em Cruz Elevado}

No teste de LCE, não foram observadas diferenças entre os grupos tratados e o controle no tempo de permanência nos braços fechados, como mostrado na Figura $3 \mathrm{~A}$, bem 
como no número de entradas nos braços fechados, apresentado na Figura 3B.

Figura 3. Efeito da administração de Dexametasona sobre o comportamento de ansiedade. (A) tempo de permanência nos braços fechados; (B) número de entradas nos braços fechados. Controle $n=13, \mathrm{DEX} 1 \mathrm{mg} / \mathrm{Kg} \mathrm{n}=15$ e DEX $10 \mathrm{mg} / \mathrm{Kg} \mathrm{n}=11$.

\section{Labirinto em Cruz Elevado}

A

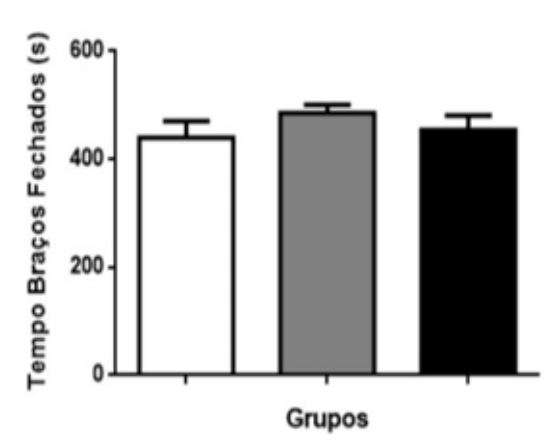

B

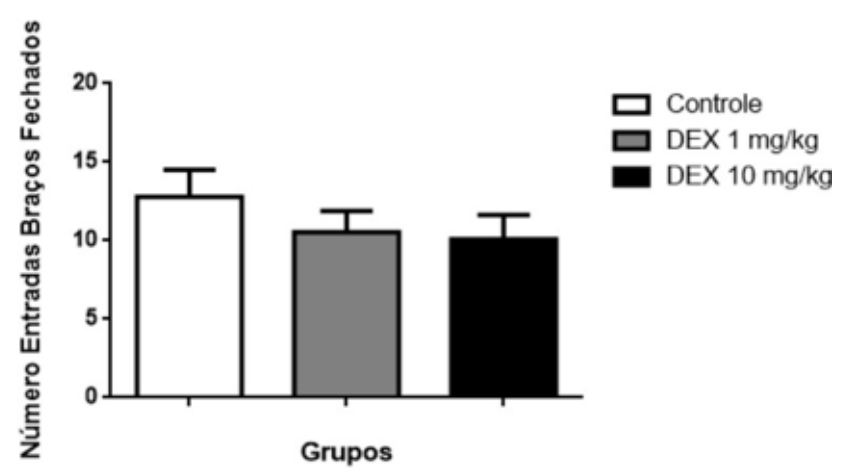

Nossos resultados mostraram que as doses $1 \mathrm{e}$ $10 \mathrm{mg} / \mathrm{kg}$ não mudaram a resposta de ansiedade do animal em 7 dias de tratamento. Embora o rato jovem apresente grande capacidade de resposta plástica, o comportamento emocional não apresentou as mudanças descritas em outros estudos.

Manojlović-Stojanoski et al ${ }^{15}$ mostraram que a administração pré-natal de DEX $0,5 \mathrm{mg} / \mathrm{Kg}$, no teste de LCE, reduziu significativamente o número de entradas nos braços abertos, o tempo de permanência nos braços abertos e a distância percorrida nos braços abertos, revelando um aumento do comportamento de ansiedade comparado aos animais controles ${ }^{15}$. Outro trabalho mostrou que a exposição 
pré-natal a DEX $0,2 \mathrm{mg} / \mathrm{Kg}$, resultou em uma redução no tempo de permanência nos braços abertos e no número de entradas nos braços abertos e, um aumento no tempo de permanência nos braços fechados no teste de LCE $^{16}$. Esses estudos indicam que o cérebro pré-natal tem grande capacidade plástica e pequenas doses da DEX podem promover mudança na ansiedade.

Corroborando com achados científicos de alguns trabalhos, o presente estudo não identificou mudanças relacionadas à ansiedade. O tratamento com DEX nas doses de 2,4 e $8 \mathrm{mg} / \mathrm{Kg}$ durante 7 dias, não provocou mudanças no comportamento semelhante à ansiedade em ratos ${ }^{17}$. Tal comportamento foi avaliado pela percentagem da entrada total nos braços abertos ou fechados do labirinto ${ }^{17}$. Outro trabalho mostrou que o tratamento com DEX $0,5 \mu \mathrm{g} / \mathrm{g}$, $0,3 \mu \mathrm{g} / \mathrm{g}$ e 0,1 $\mu \mathrm{g} / \mathrm{g}$, nos dias 1,2 e 3 de vida, respectivamente, não apresentou diferenças entre os grupos em relação ao tempo total de permanência nos braços abertos e fechados e, na plataforma central. Além disso, não encontrou diferença no número de entradas nos braços, bem como na proporção de entradas no braço aberto para entradas no braço fechado ${ }^{13}$.

\section{Teste de Labirinto em T Elevado}

O teste LTE não indicou alteração da memória espacial dos animais, não houve diferença no tempo de exploração do objeto nem no tempo de permanência e na percentagem 
de entradas no braço aberto esquerdo do labirinto, como representado na Figura 4.

Figura 4. Efeitos da administração de Dexametasona sobre a memória espacial. Controle $\mathrm{n}=10$, DEX $1 \mathrm{mg} / \mathrm{Kg} \mathrm{n}=11$ e DEX $10 \mathrm{mg} / \mathrm{Kg} \mathrm{n}=9$.

\section{Labirinto em T Elevado}

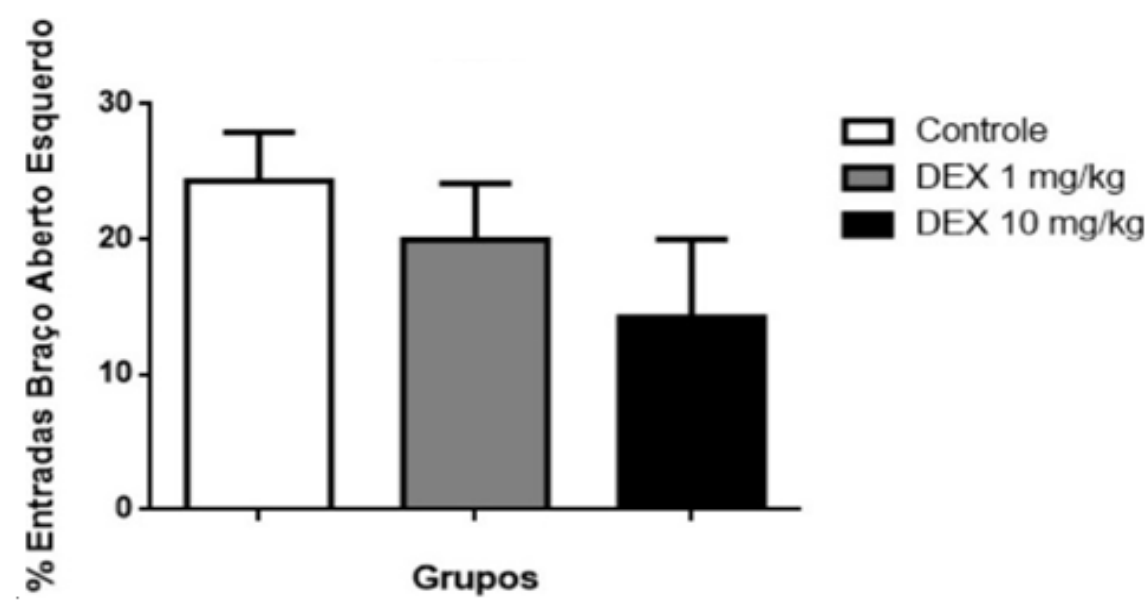

Esse resultado contraria o achado de outros estudos, foi demonstrado que os glicocorticoides podem levar a efeitos adversos no desenvolvimento do hipocampo e na memória espacial. Deficits mostrados, através do Labirinto em $\mathrm{Y}$, em animais tratados com $0,5 \mathrm{mg} / \mathrm{kg}$ de DEX a partir do $4^{\circ}$ dia pós-natal, durante 3 dias e testados no $35^{\circ}$ dia ${ }^{18}$. Embora 0 tempo de tratamento e a dose de DEX tenham sido menores com relação ao presente estudo, é possível que a plasticidade neural do cérebro pós-natal tenha promovido alterações de memória detectáveis à longo prazo. 
Onaolapo et al ${ }^{19}$ avaliaram o efeito do tratamento com DEX nas dosagens de $0,5 \mathrm{mg} / \mathrm{kg}, 1,0 \mathrm{mg} / \mathrm{kg}$ e $1,5 \mathrm{mg} / \mathrm{kg}$ durante 14 dias sobre a memória espacial, através do labirinto em $\mathrm{Y}$ elevado, após o primeiro e último dia de tratamento. No teste realizado no dia 1 , observaram um aumento significativo no desempenho da tarefa de memória espacial nas dosagens de $0,5 \mathrm{mg} / \mathrm{Kg}$ e $1,5 \mathrm{mg} / \mathrm{kg}$ em comparação ao grupo controle. O teste realizado no dia 14 mostrou um aumento significante na dose de $0,5 \mathrm{mg} / \mathrm{kg}$ e, uma redução nas tarefas de memória nas doses $1,0 \mathrm{mg} / \mathrm{Kg}$ e $1,5 \mathrm{mg} / \mathrm{kg}$ em comparação ao controle ${ }^{19}$.

A discussão sobre os efeitos da DEX sobre a memória espacial, e outros parâmetros emocionais e cognitivos, é complexa e envolve dados não apenas relacionados à dose e à idade do animal, mas também ao tempo do tratamento. Um estudo, através do labirinto aquático de Morris (LAM), que avalia memória espacial, demonstrou que animais de 35 dias de vida, tratados com DEX na dose de $0,2 \mathrm{mg} / \mathrm{Kg}$ por 5 dias consecutivos, apresentaram uma redução na latência de escape durante a fase de aprendizagem. Por outro lado, os animais tratados com a mesma dose por 7 dias, seguido de 7 dias sem tratamento e depois mais 7 dias de tratamento com DEX, apresentaram um aumento na latência de escape, nas fases de aprendizagem e memória espacial, retratando que tratamento curto com DEX promove melhor desempenho no LAM, mas o tratamento prolongado produz deficits de desempenho no mesmo teste ${ }^{20}$. 
Em um outro estudo utilizando o LAM, foi demonstrado que o tratamento com DEX nas doses de 2,4 e $8 \mathrm{mg} / \mathrm{Kg}$ durante 6 dias com essas dosagens de DEX não apresentou efeito significativo sobre a latência de escape no labirinto, como uma medida de memória espacial de longo e de curto prazo $^{17}$. Nosso estudo corrobora com os resultados desse trabalho mostrando que o tratamento com DEX não altera a memória espacial.

Alguns animais foram perdidos no teste do LCE porque pularam do aparato e no teste do LTE porque não exploraram o objeto durante os treinos e, portanto, não foram submetidos ao teste.

Embora os testes comportamentais sejam um método científico relevante para avaliação do sistema nervoso, não são as únicas ferramentas de investigação da funcionalidade neural. Análises bioquímicas podem mostrar alteração desse tecido e ativação de mecanismos de defesa e compensação. Como o presente estudo utilizou apenas os testes comportamentais na avaliação funcional dos animais, a interpretação do resultado deve levar em conta que a DEX pode ter afetado o sistema límbico, avaliado através da ansiedade pelo teste do LCE e o hipocampo, estrutura relacionada à memória espacial e avaliada pelo LTE, mas essas estruturas podem ter se reorganizado bioquimicamente e a função foi preservada. A bioquímica mostra-se uma ciência capaz de elucidar mecanismos celulares importantes na investigação do efeito da DEX sobre 
o sistema nervoso e deve ser considerada como ferramenta de investigação científica em trabalhos futuros.

\section{CONCLUSÃO}

O tratamento com dexametasona, nas doses de $1 \mathrm{e}$ $10 \mathrm{mg} / \mathrm{Kg} / \mathrm{dia}$, administrada em ratos jovens por 7 dias consecutivos, diminui a atividade exploratória espontânea, mas não altera a ansiedade e a memória espacial de ratos Wistar machos jovens.

\section{REFERÊNCIAS}

1.Abrahão JMB. Estudo clínico dos efeitos da Dexametasona e do Diazepam, associados a Bupivacaina a 0,5\% Sobre a pressão arterial e frequência cardíaca (Tese). São Paulo: Faculdade de Odontologia de Piracicaba, Universidade Estadual de Campinas, 1994, 67p. http://www.repositorio.unicamp.br/handle/REPOSIP/290770

2.Dantas KDO. Perfil de vendas de medicamentos anti-inflamatórios em farmácias comunitárias no município de Caicó - RN (Trabalho de Conclusão de Curso). Cuité: Universidade Federal de Campina Grande, 2019, 63p.

http://dspace.sti.ufcq.edu.br:8080/jspui/handle/riufcg/8320

3.Ross R, White CP. Evaluation of hydrocortisone in prevention of postoperative complications after oral surgery: a preliminary report. Oral Surg 1958;16:220-6.

4.Cella EC. Efeitos da administração de dexametasona durante o período gestacional em parâmetros de comportamento materno, emocional, memória e cognição no pós-parto de ratas (Dissertação). Florianópolis: Universidade Federal de Santa Catarina, 2018, 78p. https://repositorio.ufsc.br/handle/123456789/198836

5.Bianco AFR. Desenvolvimento de nanocarreadores para entrega combinada de cisplatina e dexametasona para o tratamento de glioblastoma multiforme (Dissertação). São Paulo: Instituto de Ciências Biomédicas, Universidade de São Paulo, 2019, 71p. https://www.teses.usp.br/teses/disponiveis/42/42136/tde-22012020102646/publico/Arthur Filipini Rodrigues Bianco Mestrado Original Parcial.pdf

6.Lin $\mathrm{HJ}$, Huang CC, Hsu KS. Effects of neonatal dexamethasone treatment on hippocampal synaptic function. Ann Neurol 2006;59:93951. http://doi.org/10.1002/ana.20885 
7.Tsai KJ, Sze CI, Lin YC, Lin YJ, Hsieh TH, Lin CH. A Single Postnatal Dose of Dexamethasone Enhances Memory of Rat Pups Later in Life. PLOS ONE 2016;11:1-18.

http://doi.org/10.1371/journal.pone.0165752

8. Nagano M, Liu M, Inagaki $H$, Kawada $T$, Suzuki $H$. Early intervention with fluoxetine reverses abnormalities in the serotonergic system and behavior of rats exposed prenatally to dexamethasone. Neuropharmacology 2012;63:292-300.

http://doi.org/10.1016/j.neuropharm.2012.03.027

9.Bondar NP, Reshetnikov VV, Burdeeva KV, Merkulova TI. Effect of neonatal dexamethasone treatment on cognitive abilities of adult male mice and gene expression in the hypothalamus. Vavilov J Gen Breeding 2019;23:456-64. http://doi.org/10.18699/VJ19.514

10.Nagano M, Ozawa $H$, Suzuki $H$. Prenatal dexamethasone exposure affects anxiety-like behaviour and neuroendocrine systems in an agedependent manner. Neurosci Res 2008;60:364-71.

http://doi.org/10.1016/j.neures.2007.12.005

11.Park DI, Kim HG, Jung WR, Shin MK, Kim KL. Mecamylamine attenuates dexamethasone-induced anxiety-like behavior in association with brain derived neurotrophic factor upregulation in rat brains. Neuropharmacology 2011;61:276-82.

http://doi.org/10.1016/j.neuropharm.2011.04.013

12.Yilmaz T, Gedikli O, Yildirim M. Evaluation of spatial memory and locomotor activity during hypercortisolism induced by the administration of dexamethasone in adult male rats. Brain Res 2015;1595:43-50. http://doi.org/10.1016/j.brainres. 2014.04.034

13.Kamphuis PJGH, Croiset G, Bakker JM, Van Bel F, Van Ree JM, Wiegant VM. Neonatal dexamethasone treatment affects social behaviour of rats in later life. Neuropharmacology 2004;47:461-74. http://doi.org/10.1016/j.neuropharm.2004.04.008

14. Yates NJ, Robertson D, Rodger J, Martin-iverson MT. Effects of Neonatal Dexamethasone Exposure on Adult Neuropsychiatric Traits in Rats. PLOS ONE 2016;11:1-23.

http://doi.org/10.1371/journal.pone.0167220

15.Manojlović-Stojanoski M, Nestorovic N, Petković B, Balind SR, Ristić $\mathrm{N}$, Trifunovic $\mathrm{S}$, et al. The effects of prenatal dexamethasone exposure and fructose challenge on pituitary-adrenocortical activity and anxietylike behavior in female offspring. Tissue Cell 2020;62:101309. http://doi.org/10.1016/J.TICE.2019.101309

16. Huang S, Dong W, Jiao Z, Liu J, Li K, Wang H, et al. Prenatal Dexamethasone Exposure Induced Alterations in Neurobehavior and Hippocampal Glutamatergic System Balance in Female Rat Offspring. Toxicol Sci 2019;171:369-84. http://doi.org/10.1093/toxsci/kfz163

17.Abdulbasit A, Michael FS, Onaopemipo AS, Abdulmusawwir AO, Aminu I, Tobechukwu AN, et al. Glucocorticoid receptor activation selectively influence performance of Wistar rats in Y-maze. Pathophysiology 2018;25:41-50.

http://doi.org/10.1016/j.pathophys.2017.12.002 
18. Alhassan AW, Bauchi ZM, Adebisi SS, Mabrouk MA, Sabo AM. The effect of early administration of glucocorticoids on learning and spatial memory. Niger J Physiol Sci 2009;24:137-9.

http://doi.org/10.4314/njps.v24i2.52915

19.Onaolapo OJ, Onaolapo AY, Akinola OR, Anisulowo TO. Dexamethasone Regimens Alter Spatial Memory and Anxiety Levels in Mice. Behav Brain Sci 2014;4:159-67.

http://doi.org/10.4236/jbbs.2014.44019

20.Gómez ABS, León HRE, Zenteno GR. Short-and long- term treatments with dexamethasone have different effects on spatial learning and memory. Clin Exp Pharmacol Physiol 2014;4:1-4. http://doi.org/10.4172/2161-1459.1000161 